\title{
Variación estacional de la composición proximal del mejillón Tagelus peruvianus (Bivalvia: Solecurtidae) del Golfo de Nicoya, Puntarenas, Costa Rica
}

\author{
Cristian Fonseca Rodríguez ${ }^{1}$, Carolina Marín-Vindas ${ }^{1}$, Fabián Chavarría-Solera $^{1}$, Rafael A. Cruz ${ }^{1}$ \\ \& Pedro Toledo Agüero ${ }^{2}$ \\ 1. Estación de Biología Marina, Escuela de Ciencias Biológicas, Universidad Nacional. Puntarenas, Costa Rica, P.O.B. \\ 1126-5400; cfonseca@una.ac.cr, cmari@una.ac.cr, fbnchs@gmail.com, rcruzsoto47@gmail.com \\ 2. Departamento de Acuicultura, Facultad de Ciencias del Mar, Universidad Católica del Norte, Chile; Centro de estu- \\ dios avanzados en zonas áridas (CEAZA); ptoledo@ucn.cl
}

Recibido 22-IX-2010. C Corregido 28-II-2011. Aceptado 31-III-2011.

\begin{abstract}
Seasonal variation in proximate composition of mussels Tagelus peruvianus (Bivalvia: Solecurtidae) from the Gulf of Nicoya, Puntarenas, Costa Rica. Marine bivalves are a very important food source for human consumption, and species that has not been of traditional use as a fishery resource are gaining interest. Seasonal variation in proximate composition, condition index and energy or caloric content of the mussel Tagelus peruvianus were studied in the Gulf of Nicoya, Puntarenas, Costa Rica. From November 2007 to October 2008, a total of 35 to 40 specimens per month were collected. The proximate composition using the AOAC methods was determined. Results showed that the condition index during December, January and May decreased, indicative of two spawning periods and one gonadal resting phase. Soft tissues were respectively characterized by protein $(61.9 \pm 4.3 \%)$, carbohydrates $(15.7 \pm 2.4 \%)$, ash $(14.0 \pm 1.9 \%)$ and lipids $(8.5 \pm 1.7 \%)$. The average caloric content was $5.0 \pm 0.1 \mathrm{kcal} / \mathrm{g}$. The results showed that the decrease in protein and fat percentage, and calories content, occurred during the spawning seasons. We suggest that T. peruvianus has an optimal nutritional value for human consumption because of the low-fat and moderate protein content. Rev. Biol. Trop. 59 (4): 1517-1523. Epub 2011 December 01.
\end{abstract}

Key words: Tagelus peruvianus, mussel, proximate composition, condition index, caloric content, Gulf of Nicoya, Costa Rica.

La demanda por alimentos ricos en proteína es cada vez mayor, especialmente en países en desarrollo y subdesarrollo, lo cual estimula la exploración de recursos no tradicionales (Heather \& Benkendorff 2008).

Los bivalvos marinos son comunes e importantes en los ecosistemas marinos y comercialmente valiosos ya que son una fuente importante de proteína, minerales y vitaminas esenciales de alto valor biológico para la población humana (Astorga et al. 2007, Fuentes et al. 2009).

Los estudios de tipo bioquímico permiten determinar el valor nutricional de cualquier organismo y proporcionan información que ayuda a entender su balance energético y ecológico (Giese \& Pearse 1974). Diversos antecedentes muestran que los valores bioquímicos de moluscos bivalvos varían entre las especies, individuos, edad, sexo, medio ambiente y época del año de la recolecta de las muestras (Pazos et al. 1997, Kang et al. 2000, Dridi et al. 2007, Liu et al. 2008, Yan et al. 2010).

La actividad metabólica en moluscos es el resultado de interacciones complejas entre la disponibilidad de alimento, condiciones ambientales, crecimiento y reproducción (Gabbott 1983). Los bivalvos marinos tienen ciclos 
estacionales de almacenamiento y utilización de energía estrechamente relacionados con la actividad reproductiva (Giese 1969, Barber \& Blake 1981).

Entre los bivalvos de la costa pacífica de Costa Rica, destaca Tagelus peruvianus Pilsbry \& Olsson 1941 (mejillón o barba de hacha). Esta especie presenta un amplio rango de distribución en la costa del Pacífica de América, desde Diggs Point, Baja California hasta Negritos, Perú (Keen 1971). La extracción de esta especie representa una actividad económica complementaria de los pescadores artesanales $\mathrm{y}$ es una de las especies de mayor pesca y consumo (Rojas et al. 1988, Cruz \& Jiménez 1994). Sin embargo, la información científica sobre este molusco se limita a estudios realizados por Rojas et al. (1988) sobre el tamaño, densidad y reproducción. Por otra parte, se han realizado estudios de la composición bioquímica de otros moluscos de Costa Rica como Báez (1985) en Polymesoda inflata; Blanco \& Montero (1992) en Strombus galeatus; Cruz \& Villalobos (1993) en Mytella guyanensis; Jiménez-Arce (1993) en Strombus gracilior y Ruiz et al. (1998) en Polymesoda radiata.

Realizar una evaluación de la composición bioquímica de $T$. peruvianus permitirá proporcionar algunos criterios sobre los periodos estacionales de extracción, utilizando un sistema sostenible de protección del recurso. El objetivo de este trabajo fue determinar la composición proximal y su relación con el índice de condición del mejillón T. peruvianus del estero de Ramas, Golfo de Nicoya, Costa Rica.

\section{MATERIALES Y MÉTODOS}

Recolecta de muestras: Mensualmente, entre noviembre 2007 y octubre 2008, se recolectaron especímenes de $T$. peruvianus $(n=35$ a 40), del banco natural del Golfo de Nicoya Puntarenas, Costa Rica (1000'29.71'" $\mathrm{N}$ - 85'52'31.12" W). Posteriormente, los ejemplares fueron medidos (longitud total) utilizando un vernier Fowler $( \pm 0.05 \mathrm{~mm}$ de precisión) y pesados en una balanza electrónica AND GX-2000 ( $\pm 0.01 \mathrm{~g}$ de precisión). El tejido blando de los individuos fue removido y drenado por 10 minutos, se registró su peso húmedo y finalmente se deshidrató en horno a $100^{\circ} \mathrm{C}$ por $24 \mathrm{~h}$. El material seco fue molido y homogeneizado manualmente con mortero para realizar los análisis químicos.

\section{Análisis químicos e índice de condición:}

El contenido de humedad se determinó según la metodología de AOAC (1984). Para cuantificar el contenido de proteína bruta se utilizó el método de Kjeldhal en un equipo LABCON$\mathrm{CO}$. Los lípidos totales fueron determinados utilizando un equipo Soxhlet (Lab-Line Instruments, Inc., ILL, USA) y extraído con éter de petróleo. Las cenizas se obtuvieron por calcinación lenta, incrementando la temperatura hasta $500^{\circ} \mathrm{C}$ por $12 \mathrm{~h}$ (AOAC 1984). Los extractos libres de nitrógeno (ELN) fueron calculados por diferencia de la suma de lípidos totales, proteína bruta y cenizas. El valor calórico del tejido blando seco de T. peruvianus se calculó utilizando los factores de conversión recomendados para moluscos (Ansell et al. 1980, Lucas $\&$ Benninger 1985).

El índice de condición de los mejillones fue calculado utilizando la siguiente relación (Ojea et al 2004, Liu et al 2008).

$$
\text { IC }=\frac{\text { peso seco del tejido blando } \times 100}{\text { peso seco de las conchas }}
$$

El tratamiento estadístico de los datos se realizó con el paquete estadístico Statistic 6.0, mediante el cual se determinaron las correlaciones (Pearson) entre los porcentajes de proteína bruta, lípidos totales, cenizas, carbohidratos e índice de condición. El supuesto de normalidad de los datos fue probado mediante la prueba de Shapiro-Wilks y la homogeneidad de la varianza fue evaluada usando la prueba de Levene (Sokal \& Rohlf 1995). Para cada parámetro evaluado se realizó un ANDEVA de una vía seguida de comparaciones múltiples de Tukey para determinar si existe diferencia significativa entre la composición bioquímica y el índice de condición durante 
los meses de muestreos con un nivel de significancia del 5\%. Los datos son reportados como promedio \pm desviación estándar.

\section{RESULTADOS}

En el Cuadro 1 se muestran los valores biométricos de $T$. peruvianus. La longitud media de los mejillones muestreados fue de $62.5 \pm 9.6 \mathrm{~mm}$, el peso total medio de $12.0 \pm 4.1 \mathrm{~g}$ y el peso fresco de $5.8 \pm 1.5 \mathrm{~g}$. La longitud máxima medida en el presente trabajo fue de $80.8 \mathrm{~mm}$, resultando muy superior a la informada por Keen (1971) de 57mm y muy similar a los $80.1 \mathrm{~mm}$ indicado por Skoglund (1991).

La variación mensual del peso seco y del IC del tejido blando del mejillón se muestra en la Fig. 1. El valor máximo obtenido para el peso seco fue de $1.70 \mathrm{~g}$ (julio) y se observan dos periodos de descenso, de noviembre

\section{CUADRO 1}

Medidas biométricas de T. peruvianus (promedio \pm desviación estándar, $\mathrm{n}=30$ ) colectados durante los meses de noviembre 2007 a octubre 2008 en el Golfo de Nicoya, Costa Rica

\section{TABLE 1}

Biometrics measurements of T. perivuanus (mean \pm sd, $n=30$ ) collected from November 2007 to October 2008 Nicoya Gulf, Costa Rica

\begin{tabular}{lccc}
\multicolumn{1}{c}{ Mes-Año } & $\mathrm{L}(\mathrm{mm})$ & $\mathrm{PT}(\mathrm{g})$ & $\mathrm{PF}(\mathrm{g})$ \\
Nov-07 & $56.7 \pm 4.9$ & $10.7 \pm 2.8$ & $6.04 \pm 1.5$ \\
Dic-07 & $56.1 \pm 3.9$ & $8.7 \pm 2.2$ & $4.32 \pm 1.0$ \\
Ene-08 & $59.6 \pm 6.1$ & $11.1 \pm 2.2$ & $5.31 \pm 1.2$ \\
Feb-08 & $58.8 \pm 9.5$ & $11.8 \pm 3.1$ & $6.01 \pm 1.2$ \\
Mar-08 & $54.7 \pm 6.0$ & $8.6 \pm 2.4$ & $4.87 \pm 1.4$ \\
Abr-08 & $61.3 \pm 13.3$ & $10.9 \pm 6.2$ & $5.81 \pm 2.9$ \\
May-08 & $70.5 \pm 11.1$ & $16.5 \pm 5.1$ & $6.70 \pm 2.0$ \\
Jun-08 & $80.8 \pm 6.6$ & $19.0 \pm 4.1$ & $8.30 \pm 1.7$ \\
Jul-08 & $81.3 \pm 9.3$ & $19.9 \pm 7.1$ & $8.66 \pm 2.7$ \\
Ago-08 & $55.9 \pm 8.1$ & $8.7 \pm 3.5$ & $4.26 \pm 1.7$ \\
Set-08 & $56.7 \pm 4.7$ & $8.9 \pm 2.5$ & $4.79 \pm 1.3$ \\
Oct-08 & $57.7 \pm 4.5$ & $8.9 \pm 2.2$ & $4.54 \pm 1.0$ \\
\hline
\end{tabular}

L: longitud de la concha, PT: peso total, PF: peso fresco de la carne.

L: shell length, PT: total weight, PF: wet weight.
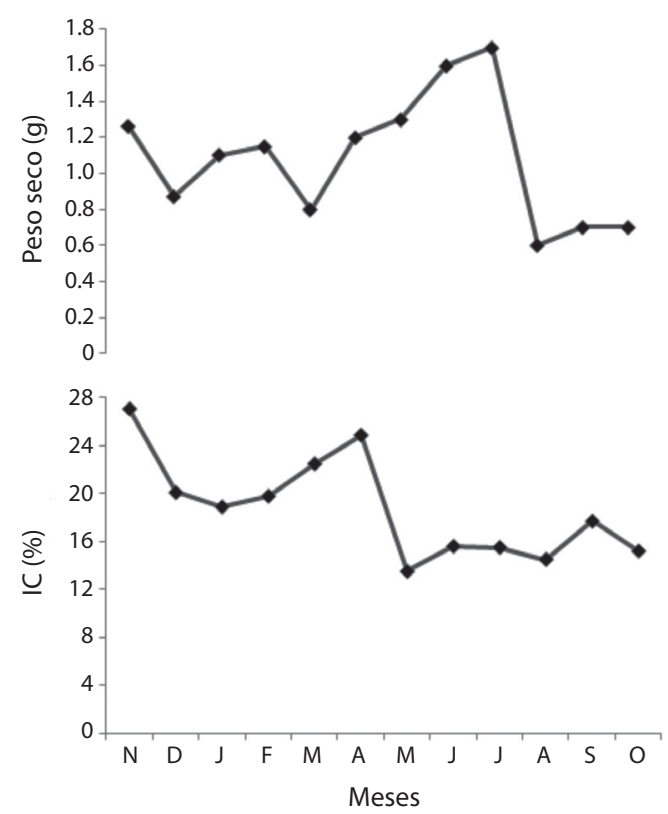

Fig. 1. Variación mensual del peso seco e índice de condición (IC) del tejido blando de $T$. peruvianus (noviembre 2007 - octubre 2008).

Fig. 1. Monthly variation of dry weight and condition index (IC) of soft tissue T. peruvianus (November 2007-October 2008).

$(1.26 \mathrm{~g})$ a marzo $(0.80 \mathrm{~g})$ y de julio $(1.70 \mathrm{~g})$ a octubre $(0.70 \mathrm{~g})$.

El patrón mensual del IC de $T$. peruvianus varió entre $13.5 \pm 2.7 \%$ y $27.1 \pm 3.3 \%$, presentando valores máximos en noviembre, marzo y abril, para decrecer significativamente $(\mathrm{p}<0.05)$. Los valores más bajos del IC se detectaron en diciembre y enero y de mayo a octubre. No se encontró una correlación entre la variación del peso seco del tejido blando y el IC (Pearson, $r=0.0344, p<0.05$ ).

Los cambios en la composición bioquímica del tejido blando de T. peruvianus durante el año se muestran en el Cuadro 2 y estan expresados en porcentaje en base seca. Las proteínas fueron el principal constituyente, presentan tres picos máximos, en noviembre $(64.47 \%)$, marzo $(61.87 \%)$ y agosto $(69.60 \%)$, con diferencias significativas respecto a los demás meses $(\mathrm{p}<0.05)$. 
CUADRO 2

Variación mensual en la composición proximal (promedio \pm desviación estándar) expresado en peso seco, de T. peruvianus, durante los meses de noviembre del 2007 a octubre del 2008 en el Golfo de Nicoya, Costa Rica

TABLA 2

Monthly variation in proximate composition (mean \pm sd), in dry matter, of T. peruvianus, from November 2007 to October 2008, Nicoya Gulf, Costa Rica

\begin{tabular}{lcccccc} 
Mes-Año & Humedad & Ceniza & Proteína bruta & Lípidos totales & ELN & Valor calórico \\
Nov-07 & $79.0 \pm 1.9$ & $11.02 \pm 0.24$ & $64.47 \pm 0.51$ & $9.85 \pm 0.17$ & 14.6 & 5.2 \\
Dic-07 & $79.8 \pm 1.0$ & $15.07 \pm 0.30$ & $60.33 \pm 0.31$ & $8.89 \pm 0.01$ & 15.7 & 4.9 \\
Ene-08 & $79.2 \pm 1.4$ & $13.76 \pm 0.51$ & $58.02 \pm 0.19$ & $9.63 \pm 0.11$ & 18.5 & 5.0 \\
Feb-08 & $81.2 \pm 2.6$ & $12.96 \pm 0.50$ & $58.94 \pm 0.70$ & $9.68 \pm 0.06$ & 18.4 & 5.0 \\
Mar-08 & $82.5 \pm 1.5$ & $13.39 \pm 0.12$ & $61.87 \pm 1.07$ & $9.85 \pm 0.18$ & 14.9 & 5.1 \\
Abr-08 & $79.4 \pm 1.3$ & $15.20 \pm 0.80$ & $57.54 \pm 0.39$ & $10.45 \pm 0.25$ & 16.8 & 4.9 \\
May-08 & $80.5 \pm 1.8$ & $16.86 \pm 0.36$ & $60.18 \pm 0.41$ & $8.27 \pm 0.53$ & 14.7 & 4.8 \\
Jun-08 & $80.3 \pm 1.6$ & $18.65 \pm 0.36$ & $56.50 \pm 0.09$ & $6.62 \pm 0.09$ & 18.2 & 4.6 \\
Jul-08 & $81.1 \pm 1.8$ & $14.87 \pm 0.23$ & $58.19 \pm 0.30$ & $6.61 \pm 0.11$ & 20.3 & 4.8 \\
Ago-08 & $85.0 \pm 1.9$ & $13.19 \pm 0.72$ & $69.60 \pm 0.46$ & $6.28 \pm 0.08$ & 10.9 & 5.0 \\
Set-08 & $85.0 \pm 1.4$ & $11.65 \pm 0.52$ & $65.83 \pm 0.52$ & $6.98 \pm 0.03$ & 15.5 & 5.0 \\
Oct-08 & $85.6 \pm 1.8$ & $13.34 \pm 0.84$ & $68.42 \pm 0.57$ & $5.45 \pm 0.10$ & 12.8 & 4.9 \\
\hline
\end{tabular}

ELN: extractos libres de nitrógeno. ELN: Nitrogen-free extracts.

Los extractos libres de nitrógeno (ELN) mostraron valores significativamente altos $(p<0.05)$ en enero, febrero, abril, junio y julio, en los meses en que la proteína presentó valores mínimos, se dio una correlación negativa entre estos componentes (Pearson, $\mathrm{r}=-0.87, \mathrm{p}<0.05$ ).

Los lípidos totales representaron la fracción más pequeña (5.45 a 10.45\%), se alcanzaron los valores más altos entre enero y abril, para luego disminuir hasta octubre, con diferencias significativas $(\mathrm{p}<0.05)$ y una correlación positiva entre el porcentaje de lípidos totales y el índice de condición (Pearson, $\mathrm{r}=0.80, \mathrm{p}<0.05)$.

Las cenizas se incrementaron desde el mes de marzo con un máximo en junio (18.65\%) y un mínimo en noviembre $(11.02 \%)$, que mostroron diferencias significativas $(\mathrm{p}<0.05)$ entre los meses de mayor y menor porcentaje.

El valor calórico promedio fue de $4.97 \pm 0.16 \mathrm{Kcal} / \mathrm{g}$, acorde con lo reportado para invertebrados acuáticos (Winberg 1971) variando mensualmente (Cuadro 2) con diferencias significativas $(\mathrm{p}<0.05)$ en noviembre, marzo y setiembre donde se observaron los máximos valores, que coincidieron con los más altos valores de lípidos totales y proteína bruta, antes de iniciarse el periodo de desove natural. Se encontró una relación inversa entre el valor calórico y el porcentaje de cenizas (Pearson, $\mathrm{r}=-0.91, \mathrm{p}<0.05)$.

\section{DISCUSIÓN}

Los nutrimentos cuantificados de T. peruvianus recolectados variaron mensualmente $\mathrm{y}$ están relacionados con su ciclo reproductivo. Similares observaciones han sido descritas para otros bivalvos como Ruditapes decussatus y Tapes philippinarum (Beninger \& Lucas 1984), Mytella guyanensis (Cruz \& Villalobos 1993), Glycymeris glycymeris (Galap et al. 1997), Ruditapes philippinarum (Ojea et al. 2005), Laternula elliptica (Ahn et al. 2003) y Fulvia mutica (Liu et al. 2008).

Debido a que el índice de condición es considerado como uno de los métodos indirectos relacionado con el desarrollo reproductivo (Ojea et al. 2004, Peharda et al. 2006), y que 
permite describir la calidad comercial y estado fisiológico de los moluscos bivalvos (Orban et al. 2002), el índice de condición obtenido en este trabajo para este bivalvo, puede ser considerado como un buen indicador del estado de su ciclo reproductivo.

El índice de condición en T. peruvianus, indica que existe actividad reproductiva durante todo el año, ya que los organismos se encuentran maduros durante noviembre, marzo y abril, con desoves de diciembre a enero y de mayo a octubre, en donde se registran los valores más bajos del IC. Resultados similares sobre periodos de desove máximo fueron encontrados por Rojas et al. (1988) para esta misma especie. El IC se mantuvo bajo durante los meses de junio a octubre, indicando que los organismos se encuentran en fase de recuperación para un nuevo ciclo de desarrollo gonadal. Esto coincide con lo reportado por Fournier (1992), Ruiz et al. (1998), Dridi et al. (2007), Mladineo et al. (2007) y Liu et al. (2008).

Los bajos porcentajes de lípidos y proteína calculados durante los periodos de desove coincidieron con los resultados obtenidos en otros estudios como los de Peiters et al. (1980), Davis \& Wilson (1983), Bressan \& Marín (1985) y Cruz \& Villalobos (1993), indicando que éstos nutrimentos son importantes constituyentes orgánicos de los ovocitos (Lucas \& Benninger 1985).

Los porcentajes de carbohidratos (ELN) varían inversamente con los de proteína y concuerda con los resultados obtenidos por Bressan \& Marín (1985), Cruz \& Villalobos (1993), Páenz-Osuna et al. (1993) y Ruiz et al. (1998).

El contenido calórico en el músculo de T. peruvianus está dado principalmente por las proteínas, las cuales se vieron afectadas durante el ciclo reproductivo $(\mathrm{p}<0.05)$, presentando valores altos justo antes del inicio de los desoves para posteriormente disminuir. Esto evidencia los altos requerimientos del mejillón durante el periodo del desarrollo gonadal, que según lo sugerido por Tyler (1973) y Shafee (1981), está muy relacionado con la formación de gametos y los procesos de desove.
El contenido de proteína en el mejillón es inferior a la carne de res, cerdo y pollo y ligeramente inferior al huevo, pero mucho mayor al de otras fuentes de proteína animal como la leche (INCAP 2007, Arenas de Moreno et al. 2000). Sin embargo, el contenido graso es muy escaso (promedio $8.5 \pm 1.7 \%$ ) y presenta ventajas comparativas frente a la recomendación generalizada de reducir el consumo de grasas.

Lo anteriormente descrito permite concluir que $T$. peruvianus presenta valores nutricionales óptimos para el consumo humano, por su bajo contenido de grasa y su aceptable contenido proteico. Se determina, además, que el índice de condición es un buen indicador del ciclo reproductivo de esta especie.

\section{AGRADECIMIENTOS}

Este trabajo es una contribución de la Universidad Nacional (Escuela de Ciencias Biológicas), por medio del proyecto 053451, "Estrategia de Manejo Participativa de las Pesquerías del Golfo de Nicoya, Costa Rica”. Se le agradece a Jorge Boza por su colaboración.

\section{RESUMEN}

La extracción del mejillón Tagelus peruvianus representa una actividad económica complementaria de los pescadores artesanales, es una de las especies de mayor pesca y consumo ya que son una fuente importante de proteína, minerales y vitaminas esenciales para la población humana. Por lo tanto, se estudió la variación estacional de su composición proximal, índice de condición y contenido energético. Mensualmente, entre noviembre de 2007 y octubre de 2008, se recolectaron de 35 a 40 especímenes. La composición proximal se determinó siguiendo la metodología recomendada por la AOAC. Los resultados mostraron que el índice de condición disminuyó en los meses de diciembre, enero y mayo, lo que indica que hay dos periodos de desove y una fase de reposo gonadal. El componente principal del mejillón es la proteína $(61.9 \pm 4.3 \%)$, seguido de carbohidratos $(15.7 \pm 2.4 \%)$, cenizas $(14.0 \pm 1.9 \%)$ y lípidos $(8.5 \pm 1.7 \%)$. El contenido calórico promedio fue de $5.0 \pm 0.1 \mathrm{kcal} / \mathrm{g}$. La disminución en los valores de proteínas, lípidos y calorías coinciden con los periodos de desove. Se puede concluir que $T$. peruvianus presenta valores nutricionales óptimos para el consumo humano, por su bajo contenido de grasa y su aceptable contenido proteico. 
Palabras clave: Tagelus peruvianus, mejillón, composición proximal, índice de condición, valor calórico, Golfo de Nicoya. Costa Rica.

\section{REFERENCIAS}

Ahn, I.Y., J. Surh, Y.G. Park, H. Kwon, K.S. Choi, S.H. Kang, H.J. Choi, K.W. Kim \& H. Chung. 2003. Growth and seasonal energetics of the Antarctic bivalve Laternula elliptica from King George Island, Antarctica. Mar. Ecol. Prog. Ser. 257: 99-110.

Ansell, A., L. Frenkield \& M. Moueza. 1980. Seasonal changes in tissue weight and biochemical composition for the bivalve Donax trunculus L. on the Algerian coast. J. Exp. Mar. Biol. Ecol. 10: 137-150.

Arenas-de Moreno, L., A. Vidal, D. Huertas -Sánchez, Y. Navas, S. Uzcátequi-Bracho \& N. Huerta-Leidenz. 2000. Análisis comparativo proximal y de minerales entre carnes de iguana, polloy res.Archivos Latinoamericanos de Nutrición 4: 409-415. (también disponible en línea: www.scielo.org.ve/scielo.php?script=sci arttext\&pid=S0004-06222000000400015\&lng=es\&n $\mathrm{rm}=$ iso $>$. ISSN 0004-0622).

AOAC. 1984. Official methods of analysis. Association of official analytical chemists. Washington D.C., EEUU.

Astorga, M., E. Rodríguez \& C. Díaz. 2007. Comparison of minerals and trace element concentration in two mollusks from the Strait of Magellan (Chile). J. Food Comp. Anal 20: 273-279.

Báez, R. 1985. Evaluación de algunos aspectos de la biología del molusco Polymesoda inflata (Philipp, 1851) (Bivalvia: Corbiculidae), en el manglar de Pochote, Puntarenas, Costa Rica. Tesis de Licenciatura, Escuela de Biología, Universidad de Costa Rica, San José, Costa Rica.

Barber, B. \& N. Blake. 1981. Energy storage and utilization in relation to gametogenesis in Argopecten irradians concentricus (Say). J. Exp. Mar. Biol. Ecol. 52: 121-134.

Beninger, P.G. \& A. Lucas. 1984. Seasonal variations in condition, reproductive activity, and gross biochemical composition of two species of adult clam reared in a common habitat: Ruditapes decussatus L (Jeffreys) and Tapes philippinarum (Adams \& Revé). J. Exp. Mar. Biol. Ecol. 79: 19-37.

Blanco, A. \& M. Montero. 1992. Composición químiconutricional de la carne de cambute, Strombus galeatus (Mesogastropoda: Strombidae). Rev. Biol. Trop. 40: 89-93.
Bressan, M. \& M. Marín. 1985. Seasonal variations in biochemical composition and condition index of cultured mussels (Mytilus galloprovincialis (LMK)) in the lagoon of Venice (North Adriatic). Aquaculture 48: 13-21.

Cruz, R.A. \& C.R. Villalobos. 1993. Monthly changes in tissue weight and biochemical composition of the mussel Mytella guyanensis (Bivalvia: Mytillidae) in Costa Rica. Rev. Biol. Trop. 41: 93-96.

Cruz, R.A \& J. Jiménez. 1994. Moluscos asociados a las áreas de manglar de la Costa Pacífica de América Central: Guía. EFUNA, Heredia, Costa Rica.

Davis, J.P. \& J.G. Wilson. 1983. Seasonal changes in tissue weight and biochemical composition of the bivalve Nucula turgida in Dublin Bay with reference to gametogenesis. Neth. J. Sea Res. 17: 84-95.

Dridi, A., M. Salah \& M. Elcafsi. 2007. Seasonal variation in weight and biochemical composition of the Pacific oyster, Crassostrea gigas in relation to their gametogenic cycle and the environmental conditions of the Bizert lagoon, Tunisia. Aquaculture 263: 238-248.

Fuentes, A., I. Fernández-Segovia, I. Escriche \& J. Serra. 2009. Comparison of physicochemical parameters and composition of mussels (Mytilus galloprovincialis Lmk.) from different Spanish origins. Food Chem. 112: 295-302.

Fournier, M. 1992. The reproductive biology of the tropical rocky oyster Ostrea iridescens (Bivalvia: Ostreidae) on the Pacific coast of Costa Rica. Aquaculture 101: 371-378.

Gabbott, P.A. 1983. Developmental and seasonal metabolic activities in marine mollusks, p 165-217. In P.W. Hochachka (ed.). The Mollusca. Environmental biochemistry and physiology. Academic, Nueva York, EEUU.

Galap, C., F. Leboulenger \& J.P. Grillot. 1997. Seasonal variations in biochemical constituents during the reproductive cycle of the female dog cockle Glycymeris glycymeris. Mar. Biol. 129: 625-634.

Giese, A.C. 1969. A new approach to the biochemical composition of the mollusks body. Oceanogr. Mar. Biol. 7: 175-229.

Giese, A. \& J. Pearse. 1974. Introduction general principles, p 1-41. In A.C. Giese \& J.S. Pearse (eds.). Reproduction of marine invertebrates. Academic, Nueva York, EEUU.

Heather, S. \& K. Benkendorff. 2008. The impact of diet on the growth and proximate composition of juvenile 
whelks, Dicathais orbita (Gastropoda: Mollusca). Aquaculture 276: 162-170.

Instituto de Nutrición de Centro América y Panamá. 2007. Tabla de composición de alimentos de Centroamérica. INCAP. M.T. Menchú \& H. Méndez (eds.). INCAP/OPS, Guatemala.

Jiménez-Arce, G. 1993. Composición químico-nutricional en diferentes tallas y sexos del cambute Strombus gracilior (Mesogastropoda: Strombidae) de Playa Panamá, Costa Rica. Rev. Biol. Trop. 41: 345-349.

Kang, C.K., M.S. Park, P.Y. Lee, W.J. Choi \& W.C. Lee. 2000. Seasonal variations in condition, reproductive activity, and biochemical composition of the oyster, Crassostrea gigas (Thunberg), in suspended culture in two coastal bays of Korea. J. Shellfish Res. 19: $771-778$

Keen, M. 1971. Sea shells of Tropical West America. Marine mollusks from Baja California to Peru. Stanford University, Stanford, California, EEUU.

Liu, W., Q. Li, Y. Yuan \& S. Zhang. 2008. Seasonal variations in reproductive activity and biochemical composition of the cockle Fulvia mutica (Reeve) from the eastern coast of China. J. Shellfish Res. 27: 405-411.

Lucas, A., P.G. Benninger. 1985. The use of physiological condition indices in marine bivalve aquaculture. Aquaculture 44: 187-200.

Mladineo, I., M. Peharda, S. Orhanovic, J. Bolotin, M. Pavela-Vrančić \& B.Treursić. 2007. The reproductive cycle, condition index and biochemical composition of the horse-bearded mussel Modiolus barbatus. Helgol. Mar. Res. 61: 183-192.

Ojea, J., A.J. Pazos, D. Martínez, S. Novoa, J.L. Sánchez \& M. Adad. 2004. Seasonal variation in weight and biochemical comparison of the tissues of Rudiatapes decussatus in relation to the gamatogenic cycle. Aquaculture 238: 451-468.

Ojea, J., D. Martínez, S. Novoa \& A. Cerviño-Otero. 2005. Ciclo gametogénico de una población de almeja japonesa Ruditapes philippinarum (Adams \& Reeve, 1850) en la ría de Camariñas (noroeste de España) y relación con la composición bioquímica mayoritaria. Bol. Inst. Esp. Oceanogr. 21: 337-342.

Orban, E., G. Di Lena, T. Nevigato, I. Casini, A. Marzetti \& R. Caproni. 2002. Seasonal changes in meat content, condition index and chemical composition of mussels (Mytilus galloprovincialis) cultured in two different Italian sites. Food Chem. 77: 57-65.
Páenz-Osuna, F., H. Zozueta-Padilla \& J. Osuna-López. 1993. Biochemical composition of the oysters Crassostrea iridescens (Hanley) and Crassostrea corteziensis (Hertlein) in the north-west coast of México: Seasonal changes. J. Exp. Mar. Biol. Ecol. 170: 1-9.

Pazos, A.J., G. Román, C.P. Acosta, M. Abad \& J.L. Sánchez. 1997. Seasonal changes in condition and biochemical composition of the scallop Pecten maximus L. from suspended culture in the Ria de Arousa (Galicia, N.W. Spain) in relation to environmental conditions. J. Exp. Mar. Biol. Ecol. 211: 169-193.

Peharda, M., I. Mladineo, J. Bolotin, L. Kekez \& B. Skaramuca. 2006. The reproductive cycle and potential protandric development of the Noah's Ark shell, Arca noae L.: implications for aquaculture. Aquaculture 252: 317-327.

Peiters, H., J.H. Klytmans, D.I. Zandee \& G.C. Caddé. 1980. Tissue composition and reproduction of Mytilus edulis in relation to food availability. Netherl. J. Sea Res. 14: 349-361.

Rojas, J., C. Villalobos, F. Chartier \& C. Villalobos. 1988. Tamaño, densidad y reproducción de la barba de hacha, Tagelus peruvianus (Bivalvia: Solecurtidae) en el estero de Puntarenas, Costa Rica. Rev. Biol. Trop. 36: 479-483.

Ruiz, E., J. Cabrera, R.A. Cruz \& J. Palacios. 1998. Composición bioquímica de la carne de Polymesoda radiata (Bivalvia: Corbiculidae) en Costa Rica. Rev. Biol. Trop. 46: 646-653.

Shafee, M.S. 1981. Seasonal changes in the biochemical composition and caloric content of the blanck scallop Chlamis varia (L) from Lanveos, Bay of Brest. Oceanol. Acta 4: 331-341.

Skoglund, C. 1991. Additions to the Panamic Province Bivalve (Mollusca) Literature 1971 to 1990 . The Festivus 22: 1-74.

Sokal, R.R. \& F.J. Rohlf. 1995. Biometría. Principios y métodos estadísticos en la investigación biológica. $\mathrm{H}$. Blume, Madrid, España.

Tyler, A. 1973. Caloric values of some North Atlantic Invertebrates. Mar. Biol. 19: 258-261.

Winberg, G.G. 1971. Methods for the estimation of production of aquatic animals. Academic, Nueva York, EEUU.

Yan, H., Q. Li, R. Yu \& L. Kong. 2010. Seasonal variations in biochemical composition and reproductive activity of Venus Clam Cyclina sinensis (Gmelin) from the yellow River Delta in Northern China in Relation to Environmental Factors. J Shellfish Res. 29: 91-99. 
